\title{
Inducción de la formación de moco gástrico por sangre de grado (Croton palanostigma) ${ }^{*}$
}

\author{
MIGUEL SANDOVAL, SALOMÓN AYALA, RAQUEL ORÉ, JORGE ARROYO \\ Centro de Investigación de Bioquímica y Nutrición “Alberto Guzmán Barrón”. \\ Facultad de Medicina. Universidad Nacional Mayor de San Marcos.
}

\begin{abstract}
RESUMEN
OBJETIVO: Determinar si la sangre de grado de Croton palanostigma induce a la formación de moco gástrico, como efecto protector. MATERIAL Y MÉTODOS: Se usó ratas albinas machos adultos, entre 200 a 250 g de peso, distribuidas en 4 grupos: (I) control con solución salina; (II) croton gástrico $0,8 \mathrm{~mL} / \mathrm{kg}$; (III) croton duodenal $0,8 \mathrm{~mL} / \mathrm{kg}$; (IV) ranitidina $50 \mathrm{mg} / \mathrm{kg}$; en cada grupo 10 animales. Una hora después, bajo anestesia con éter etílico, se realizó ligadura pilórica por laparatomía abdominal y una descarga de histamina para estimular la secreción. Se extrajo los estómagos y midió en la porción glandular la formación de moco por el método de Corne modificado, expresado como $\mu \mathrm{g}$ de alcian blue $/ \mathrm{mL} / \mathrm{g}$ de tejido. RESULTADOS: La producción de moco por grupos fue: control 34,5 $\pm 5,5$; croton gástrico 45,8 $\pm 12,2$; croton duodenal 50,6 $\pm 13,9$; ranitidina 39,0 $\pm 7,1$. Hay diferencia significativa de mayor formación de moco en los grupos croton gástrico y duodenal que en el control, $\mathrm{p}<0,01$; no hubo diferencia entre el grupo ranitidina y el grupo control. El grupo que tuvo mayor inducción fue el de croton por vía duodenal, lo que indicaría que su mecanismo de acción no es únicamente tópico, lo que promueve nuevos estudios. CONCLUSIONES: Encontramos inducción de formación de moco gástrico tras la aplicación de sangre de grado de croton palanostigma por vía gástrica y duodenal, pudiendo ser éste parte del mecanismo de acción de protección de la sangre de grado.
\end{abstract}

Palabras clave: Mucosa gástrica; experimentación de medicamentos; investigación.

\section{INDUCTION OF GASTRIC MUCUS BY DRAGON'S BLOOD (Croton palanostigma) ABSTRACT}

OBJECTIVE: To determine if dragon's blood Croton palanostigma induces gastric mucus secretion as a protective effect. MATERIAL AND METHODS: Adult male albine 200 to 250 $\mathrm{g}$ rats were distributed in 4 groups: (I) control with saline solution; (II) gastric croton $0,8 \mathrm{~mL} /$ $\mathrm{kg}$; (III) duodenal croton $0,8 \mathrm{~mL} / \mathrm{kg}$; (IV) ranitidine $50 \mathrm{mg} / \mathrm{kg} ; 10$ animals in each group. One hour later, pyloric ligation by abdominal laparatomy was performed under ethil ether anestesia and a histamine discharge was done to stimulate secretion. Stomachs were removed and at the glandular portion mucus secretion was measured by modified Corne method expressed as $\mu \mathrm{g}$ of alcian blue/mL/g of tissue. RESULTS: Mucus production by groups was: control 34,5 $\pm 5,5$; gastric croton $45,8 \pm 12,2$; duodenal croton $50,6 \pm 13,9$; ranitidine $39,0 \pm 7,1$. There was significative more mucus production in the gastric and duodenal croton groups than in the control group $\mathrm{p}<0,01$; there was no difference between ranitidine and control groups. Best croton induction was via duodenum, indicating that mechanism is not solely topical, to be confirmed in future studies. CONCLUSIONS: We found induction of gastric mucus production following application of dragon's blood croton palanostigma via stomach and duodenun and this could be part of the croton's protection mechanism.

Key words: Gastric mucosa; drug experiment; research.

* El resumen del presente trabajo fue publicado en Anales de la Facultad de Medicina, Volumen 63, Suplemento 2002.

Correspondencia:

Dr. Miguel Sandoval Vegas

Facultad de Medicina, UNMSM. Av. Grau 755. Lima 1, Perú

E-mail: msandovalv@sanfer.unmsm.edu.pe 


\section{INTRODUCCIÓN}

La sangre de grado, látex del Croton palanostigma, es un producto de nuestra amazonía usado por etnias nativas y difundido en diversas regiones del Perú, así como en otras latitudes; pues es un producto natural que ha traspasado las fronteras como una medicina natural efectiva. Se le conoce de diferentes maneras, como sangre de grado, sangre de drago o sangre de dragón. Se le usa de manera curativa, como cicatrizante, aplicándolo directamente sobre la herida en la piel, en mordeduras de arañas, en abrasiones y ampollas. Se ha observado en la medicina tradicional, su efecto sobre la inflamación y edema. Su mayor utilidad, muy difundida, es usándolo en las gastritis y úlceras gástricas, así como un coadyuvante en el tratamiento de las infecciones intestinales $\left(^{1-3}\right)$.

La sangre de grado o sangre de dracón u otros nombres populares es como se le conoce a la savia del árbol del Croton palanostigma, existiendo además otras variedades como Croton lechleri, Croton urucurama, Croton echinocarpus y Croton dracónico, cuyas propiedades medicinales son semejantes. Es un árbol de tallo elevado, sin ramificaciones a media altura, únicamente en copa. El nombre de la savia se debe al parecido con la sangre humana. Para obtenerla se practica incisiones transversales sobre la corteza, por donde fluye y se recolecta en recipientes en el extremo terminal del corte $\left({ }^{1-3}\right)$. La sangre de grado, es una resina de sabor astringente, está compuesta por sustancias diversas, como heterósidos, tanino, ácido benzoico, celulosa, resina dragocoresina compuesta por ésteres de alcohol resínicos- y ácido benzilacético y alcaloides, entre los que resalta la taspina $\left({ }^{1}\right)$.

Se conoce de sus propiedades protectoras frente a la injuria aguda de la mucosa gástrica $\left({ }^{4-6}\right)$, así como su poder cicatrizante, habiéndose estudiado principalmente $C$. Lechleari $\left({ }^{7,8}\right)$. En la actualidad existe la tendencia a rescatar las bondades de los productos naturales en el tratamiento de diversas enfermedades y, entre ellas, las del aparato digestivo, que se encuentra entre los cinco principales registros de defunciones en el Perú $\left({ }^{9}\right)$.

Existen estudios de otras plantas, como las de Musa spientum (plátano) y Brassicca oleracea (col) $\left({ }^{10,11}\right)$ que son usadas actualmente como antiulcerosas (gafarnate) $\left({ }^{12}\right)$, así como por el efecto protector de la mucosa gástrica de la sangre de grado. En nuestro medio no hemos encontrado explicación de cómo puede ser el mecanismo de acción, específicamente del Croton palanostigma. El objetivo de nuestra investigación es determinar si la sangre de grado de Croton palanostigma induce a la formación de moco gástrico, como efecto protector.

\section{MATERIAL Y MÉTODOS}

Como material vegetal, se usó la resina del árbol de Croton palanostigma, denominada tradicionalmente como "sangre de grado". Pertenece a la familia Euphorbiaceae; se lo obtuvo un mes antes del experimento, teniendo como procedencia el departamento de San Martín (Perú). Fue conservada entre 8 a $10^{\circ} \mathrm{C}$ en un recipiente estéril y de color caramelo.

Como material farmacológico, se empleó una solución estéril de ranitidina, bajo la forma de ranitidina base clorhidrato, como compuesto de comparación. Así mismo, se utilizó solución estéril de cloruro de sodio al $9 \mathrm{~g} / \mathrm{L}$, como control. Este material farmacológico fue de uso comercial.

Como material animal, fueron usadas un total de 40 ratas albinas machos, entre 200 a 250 g, obtenidas en el bioterio del Instituto Nacional de Salud.

Y como material químico, todos los compuestos químicos usados en la determinación de moco gástrico, fueron grado q.p. (químicamente puros).

Los animales fueron divididos aleatoriamente en cuatro grupos, cada uno con 10 animales. Los grupos fueron: (I) grupo control, a los que 
se les administró $2 \mathrm{~mL}$ de solución estéril de cloruro de sodio $9 \mathrm{~g} / \mathrm{L}$; (II) grupo croton gástrico, a los que se administró por vía cánula gástrica, la solución de sangre de grado de Croton palanostigma $0,8 \mathrm{~mL} / \mathrm{kg}$ de peso; (III) grupo croton duodenal, a los que se administró mediante inyección por vía duodenal la solución de sangre de grado de Croton palanostigma $0,8 \mathrm{~mL} / \mathrm{kg}$ de peso y (IV) el grupo ranitidina, a quienes se les administró este fármaco $50 \mathrm{mg}$ / $\mathrm{kg}$ de peso. En cada caso el volumen administrado fue $2 \mathrm{~mL}$, completándose en caso necesario con solución salina fisiológica estéril.

Todos los animales fueron puestos en ayunas de alimentos sólidos durante 24 horas antes de la experimentación. Tras la aplicación del cloruro de sodio, la sangre de grado o la ranitidina, se esperó una hora y se realizó una laparatomía abdominal, bajo anestesia de éter líquido expuesto al ambiente en cámara cerrada, para realizar una ligadura pilórica. Posteriormente se aplicó por vía subcutánea histamina $50 \mathrm{ug} / \mathrm{kg}$, descarga para estimular la secreción gástrica.

Después de dos horas de la ligadura pilórica, se realizó la gastrectomía, nuevamente bajo anestesia con éter; fue pinzado el cardias y el píloro, se extrajo el estómago y se mantuvo en una superficie entre 2 a $4{ }^{\circ} \mathrm{C}$. Se colectó el contenido gástrico, al cual se le midió el volumen y pH por método potenciométrico, con calibración del equipo con soluciones estándares de pH 4 y 7. Los estómagos fueron abiertos por la curvatura mayor, se les lavó suavemente con solución salina estéril y extrajo la porción glandular, la cual fue pesada en una balanza analítica eléctrica y se realizó posteriormente la determinación del moco gástrico.

El tejido gástrico fue colocado en $7 \mathrm{~mL}$ de solución de alcian blue $0,1 \mathrm{~g} \%$ por 2 horas, se realizó dos lavados con solución de sucrosa 0,25 $\mathrm{mol} / \mathrm{L}$, dejando el tejido en la primera lavada 15 minutos y en la segunda 45 minutos, eliminando en cada caso la solución de lavado. Posteriormente, se agregó $7 \mathrm{~mL}$ de cloruro de magnesio y dejo actuar 2 horas, realizando mo- vimientos de agitación cada 30 minutos, para realizar la extracción del moco gástrico. El líquido fue centrifugado con éter dietílico puro, para la compactación del moco. La extracción del colorante adherido al moco se realizó por solubilidad en $5 \mathrm{~mL}$ de solución etanólica al $70 \%$ $\mathrm{V} / \mathrm{V}$, separando posteriormente el colorante en el sobrenadante por centrifugación diferencial. La concentración del alcian blue, retenido por el moco y obtenido en la solución alcohólica, es proporcional a la cantidad de moco presente en la muestra. Se cuantificó por espectrofotocolorimetría realizando las lecturas a $598 \mathrm{~nm}$, expresando la concentración como $\mu \mathrm{g}$ de alcian blue $/ \mathrm{mL} / \mathrm{g}$ de tejido.

La evaluación estadística se realizó con el método de la prueba de $t$ de Student de la diferencia, aplicándose un nivel de significancia estadística para un valor de $p \leqslant 0,05$.

\section{RESULTADOS}

La medición del volumen del contenido gástrico, fue muy variado, por lo que no encontramos diferencia estadísticamente significativa (Tabla 1). La valoración del pH, mostró un incremento esperado en el grupo de animales sometidos a la acción de la ranitidina $(\mathrm{pH}$ $=2,54 \pm 0,42$ ), respecto al grupo control. Los valores de $\mathrm{pH}$ entre los grupos tratados con sangre de grado, sea por vía gástrica o duodenal,

Tabla 1.- Volúmenes del jugo gástrico de ratas tratadas con sangre de grado de Croton palanostigma gástrico y duodenal.

\begin{tabular}{lcccc}
\hline Jugo gástrico & Control & Ranitidina & Corton G & Croton D \\
\hline Promedio (mL) & 4,78 & 7 & 6,93 & 5,82 \\
DE & 2,42 & 2,19 & 2,06 & 2,30 \\
$\mathrm{n}$ & 10 & 10 & 10 & 10 \\
$p$ & & $>0,05$ & $>0,05$ & $>0,05$ \\
\hline
\end{tabular}

Croton G: Tratamiento con sangre de grado por vía gástrica. Croton D: Tratamiento con sangre de grado por vía duodenal DE: Desviación estándar. 
Tabla 2.- $\mathrm{pH}$ del jugo gástrico de ratas tratadas con sangre de grado de Croton palanostigma gástrico y duodenal.

\begin{tabular}{lcccc}
\hline Jugo gástrico & Control & Ranitidina & Corton G & Croton D \\
\hline Promedio pH & 1,49 & 2,54 & 1,67 & 1,42 \\
DE & 0,28 & 0,42 & 0,38 & 0,13 \\
n & 10 & 10 & 10 & 10 \\
$p$ & & $<0,05$ & $>0,05$ & $>0,05$
\end{tabular}

Croton G: Tratamiento con sangre de grado por vía gástrica. Croton D: Tratamiento con sangre de grado por vía duodenal DE: Desviación estándar.

no mostraron diferencia entre sí ni con el grupo control (Tabla 2).

La formación de moco gástrico fue significativamente mayor en los grupos tratados con sangre de grado que en el control observándose significancia estadística $(p \leqslant 0,05)$. El grupo de animales con croton gástrico se presentó mayor moco gástrico en un $32,7 \%$ más que el grupo control y para el caso de los animales tratados con sangre de grado por vía duodenal, la cantidad de moco gástrico fue mayor aún, alcanzando una producción de moco de $46,8 \%$ más que el control y equivalente a una producción de 10,6\% más que el moco gástrico formado por aplicación de croton gástrico. La cantidad de moco formado en los animales tratados con ranitidina, fue discretamente mayor, en $13,2 \%$, que el control (Tabla 3 ), pero sin mostrar diferencia significativa.

Tabla 3.- Moco gástrico de ratas tratadas con sangre de grado de Croton palanostigma gástrico y duodenal.

\begin{tabular}{lcccc}
\hline Jugo gástrico & Control & Ranitidina & Croton G & Croton D \\
\hline Promedio & 34,48 & 39,03 & 45,76 & 50,65 \\
DE & 5,49 & 7,12 & 12,18 & 13,93 \\
n & 10 & 10 & 10 & 10 \\
$\%$ incremento & & 13,2 & 32,7 & 46,8 \\
$p$ & & $>0,05$ & $<0,05$ & $<0,01$
\end{tabular}

Croton G: Tratamiento con sangre de grado por vía gástrica. Croton D: Tratamiento con sangre de grado por vía duodenal DE: Desviación estándar.

\section{DISCUSIÓN}

La propiedad cicatrizante de heridas y efectos antiinflamatorios de la sangre de grado de Croton palanostigma, Croton lechleari y Croton draconoides, han sido demostradas experimentalmente y los estudios señalan a una fracción alcaloide, la taspina, como la responsable de tales efectos $\left({ }^{13,14}\right)$. En este estudio demostramos el efecto de la sangre de grado del árbol Croton palanostigma, sobre la mucosa gástrica, tras la administración gástrica de $0,8 \mathrm{~mL} / \mathrm{Kg}$, acción de manera tópica, puesto que la resina estuvo en contracto directo con la mucosa; esto indicaría que este producto natural induce a la mayor formación de la barrera muco protectora contra la acción irritante del ácido clorhídrico presente en la secreción gástrica, moco cuyo $\mathrm{pH}$ referido por la literatura llega a ser hasta de 7 en las zonas muy cercanas al borde libre de las células del epitelio gástrico, favoreciendo de esta manera la protección de dicho tejido, como lo han demostrado ya trabajos anteriores en nuestro medio $\left({ }^{6}\right)$.

Por otro lado, en nuestros resultados hemos observado que la aplicación de la sangre de grado de Croton palanostigma por vía duodenal, indujo también a formación de moco gástrico y en mayor cantidad, lo que sugiere que entre sus componentes existen uno o más principios que se absorben y actúan ya no de manera tópica, es decir por la zona apical de la mucosa, sino que la acción estaría produciéndose además por la zona basal del tejido epitelial, es decir, que podría realizarse la inducción de la formación de moco que recubre al estómago por vía sistémica; este planteamiento, sujeto a posteriores demostraciones, podría ser la explicación del efecto que tiene la sangre de grado como un producto natural que permite "curar" la gastritis y las úlceras gástricas.

Está ya documentado el efecto cicatrizante de la sangre de grado $\left({ }^{7}\right)$, así como el efecto de la taspina sobre la migración celular y síntesis de colágeno, también demostrados $\left({ }^{15,16}\right)$. Sin embargo, para en el tratamiento de la gastritis y 
úlceras gástricas, además de los efectos anteriormente citados, debe contarse con una protección local externa, es decir, una protección de la zona irritada o ulcerada en el estómago, por lo que la función del moco explicaría tal efecto.

Estudios realizados con fármacos de acción protectora de la mucosa gástrica, como el sucralfato, frente a la acción agresiva de varios agentes $\left({ }^{17,18}\right)$ indican que parte de este mecanismo de protección incluye la formación de una "barrera protectora" en la mucosa lesionada, lo que conlleva señalar a la mayor formación del moco, como parte de esta acción protectora; efecto que planteamos, a la luz de nuestros resultados, podría ser parte del mecanismo de acción de citoprotección, inducida por la sangre de grado.

Las prostaglandinas también tienen un rol muy importante en la actividad de la mucosa gástrica. Diversos estudios muestran la actividad protectora de las prostaglandinas, como la $\mathrm{PGE}_{1}, \mathrm{PGE}_{2}, \mathrm{PGF}_{2 \beta}$, mediante la inducción de formación de moco, señalando acción citoproctectora, mediada por moléculas no proteicas con uniones sulfidrilo $\left({ }^{19,20}\right)$.

La acción farmacológica de la inducción de formación de moco gástrico como parte del mecanismo que favorece la regeneración del tejido irritado o ulcerado, ha sido también estudiada; está acción mucoprotectora ha sido demostrada en el uso de los antiácidos $\mathrm{Al}(\mathrm{OH}) 3$, Mylanta II y Maalox, los cuales, entre sus acciones, revelan un incremento luminal de prostaglandina E2, estimulación a células de la mucosa en las que se observó mayor vacuolización, evidencia de una mayor actividad secretora y mayor actividad mitótica $\left({ }^{21}\right)$.

Además de la secreción de moco, existen otros factores defensivos de la mucosa gástrica, como son el flujo sanguíneo y la presencia de bicarbonato en el moco, que neutraliza la acción de los iones $\mathrm{H}^{+}$del medio gástrico. Todos ellos son mecanismos diferentes al de la inhibición de la secreción ácida, como lo realiza la ranitidina. La disminución o carencia de moco, así como la disminución del flujo circulatorio, producen un efecto de retrodifusión de $\mathrm{H}^{+}$al tejido, provocando irritación y ulceración y, cuando hay protección, dilución, tamponamiento o eliminación del exceso de acidez, no hay daño tisular o éste es mínimo $\left({ }^{22}\right)$. Respecto al pH, debemos denotar que la aplicación de sangre de grado de Croton palanostigma no originó variaciones significativas entre los animales a los que se les administró sangre de grado por vía gástrica $(\mathrm{pH} 1,67)$ o duodenal $(\mathrm{pH} 1,42)$, respecto al grupo control $(\mathrm{pH} \mathrm{1,49)}$. Demostró de esta manera que no tiene acción antiácida y que no sólo la disminución de la acidez del medio gástrico es un factor que conlleve a la protección de la mucosa gástrica, sino que también se da por la formación y calidad de la barrera protectora dada por el moco; evidentemente como parte de la acción cicatrizante, antiinflamatoria y antiulcerosa que tiene la sangre de grado. Comparativamente, el grupo con ranitidina, cuya acción farmacológica es la inhibición competitiva al bloquear los receptores $\mathrm{H} 2$, se evidenció en nuestros resultados al obtener en el grupo de animales tratados con este fármaco, un promedio de $\mathrm{pH} 2,54$ y una producción de moco gástrico menor que en los grupos con croton.

En conclusión, a partir de nuestras condiciones experimentales, encontramos inducción de formación de moco gástrico tras la aplicación de sangre de grado de croton palanostigma por vía gástrica y duodenal, pudiendo ser esta parte del mecanismo de acción de protección de la sangre de grado.

\section{AGRADECIMIENTO}

Este trabajo se realizó con el apoyo del Consejo Superior de Investigaciones de la Universidad Nacional Mayor de San Marcos; Estudio 010104191.

\section{BIBLIOGRAFÍA}

1. Arellano P. El libro verde. Guía de recursos terapéuticos vegetales. Ministerio de Salud. Lima, 1992; 33,46,53. 
2. Estrella E. Plantas medicinales amazónicas. Tratado de Cooperación Amazónica. Lima Perú 1995; 210,212.

3. Barriga R. Plantas útiles de la Amazonía peruana. CONCYTEC, Lima Perú, 1994.

4. Tapia SB, Principe H, Rachumi M. Estudio controlado del efecto preventivo y terapéutico de Croton palanostigma, Aloe vera y Omepraszol, sobre lesiones gástricas inducidas por etanol en ratas albinas. Congreso Peruano de Gastroenterología. Lima, 1996.

5. Málaga E. Efecto del clorhidrato de taspina sobre la curación de úlcera gástrica inducida en ratas. Tesis de Bachiller en Biología. Lima, Univ. Peruana Cayetano Heredia.

6. Ayala S, Díaz D, Palomino M, Armas S, Paz J. Efecto protector de Croton palanostigma y Aloe vera frente a la injuria aguda de mucosa gástrica inducida por etanol en ratas. Anales de la Facultad de Medicina, UNMSM. 1999; 60(1): 22-9.

7. López-Neira L. Elaboración de una farmacéutica de aplicación tópica con efecto cicatrizante a partir del extracto atomizado del látex de sangre de drago. Tesis Químico Farmacéutico. Lima -Perú. 1999.

8. Lapa AJ. I curso Iberoamericano de validasao de plantas medicinais utilizadas nos disturbios gatrointestinais, CYTED 1998.

9. INEI. Instituto Nacional de Estadística e Informática. Perú compendio estadístico 2001. Sistema Nacional de Estadística. Lima Perú. 2001.

10. Sanyal AK, Das PK, Sinha S, Sinha YH. Banana and gastric secretion, J Pharm Pharmacology 1961; 13: 318-9.

11. Sanyal AK, Burnerjee CR, Das PK. Studies on peptic ulceratrion - part II role of banana in restraint and pregnisolone induced ulcer in albino rats. Arch Intern Pharmacodynamie Ther 1965;155: 244-8.

12. Adami E, Marzzi-Uberti E, Turba C. Pharmacological reserch on gafarmate, a new synthetic isopenoid with an antiulcer action. Arch intern Phasmacodynamie Ther 1964; 147: $113-45$.
13. Vaisberg AJ, Milla M, Planas MC, Córdova JL, Agusti ER, Ferreira R, Nustiga MC, Carlin L, Hammon GB. Taspine is cicatrizant principle in sangre de grado extracted for Croton Lechleri, Planta Med 1994; 60(6): 541-5.

14. Milla ME. Estudio sobre el mecanismo de acción del principio activo taspina de sangre de grado. Tesis Bachiller en Biología. Univ. Peruana Cayetano Heredia. Lima 1985.

15. Goñi Morgan MA. Efecto del alcaloide taspina de la sangre de grado sobre la migración celular y síntesis de colágeno en células 3T3. UPCH. Lima, 1987

16. Pieters L, De Bruyne T, Claeys M, Vlietinck A, Calomme M, Vanden Berghe D. Isolation of a dihidrobenzofuran lignan from south American dragon's blood (croton ssp) as an inhibitor of cell proliferation. University of Antwerp, Belgium, J Natural Products 1993; 56(6); 899-906.

17. Harrington SJ, Schelegel JF, Code CF. The proctective effect os sucraltate on the gastric mucosa of rats. J Clin Gastroenterol 1981; 3: 129-34.

18. Nagashima R, Hashino E, Hinohara Y, Sakai K, Haa S, Nakano H. Effect of sucralfate on etanol induced gastric munosal damage in the rat. Scand J Gastroenterol 1983; 3 (suppl 83): 17-20.

19. Szabo S, Trier JS, Frankel JW. Sulfhydryl compounds may mediate gastric cytoprotection. Science 1981; 214(9): 200-2.

20. Tarnawski A, Hollander D, Gergely, Krause WJ. Protection by prostaglandin of gastric mucosa against thermal (boiling water) injury factor fiction . (Res) VA Med Gastro 1979; 77: 433.

21. Tarnawski A, Hollander D, Cummings D, Krause WJ, Zipser RD. Are antacids acid neutralizers only, histologic, ultraestructural and functional changes in normal gastric mucosa induced by antacid. Gastroenterology 1984; 86 (5) part 2: 1276.

22. Esplugues JV, Martínez-Cuesta MA, Moreno L, Calatayud S, Beltrán B. Mecanismos defensivos de la mucosa gástrica: bases funciones y actuación farmacológica. Gastroenterol Hepatol 1997; 20(4). 